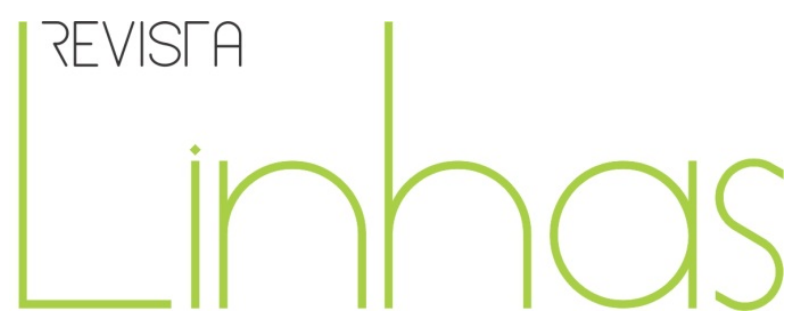

\title{
Condiciones y premisas para hacer realidad una utopia
}

\section{Resumen}

En el presente artículo se destaca que el debate en el campo de la educación de las personas con algún tipo de deficiencia o déficit biológico, no es la polémica Escuela Especial, integración escolar o inclusión. Se insiste en lo que parecen ser las verdaderas condiciones y premisas que no se cumplen o, nunca se han cumplido plenamente, en un modelo organizativo u otro, en particular y que se constituyen en las verdadera causas del por qué, estas personas no logran los aprendizajes de calidad que pueden lograr y con ellos los desarrollos correspondientes. Son las condiciones sociales y culturales e incluso como parte de ellas, las políticas e ideológicas las verdaderas causas que hacen que los niños y niñas con deficiencias no aprendan y se desarrollen en un modelo educacional u otro. Se insiste en que esta lucha o debate existe desde la época de la antigüedad, pasando por diferentes momentos y que el actual es apena uno de ellos y que aunque se ha avanzado, siguen presentándose las consecuencia de deficiente educación, que no facilita los aprendizajes, el desarrollo integral de estas personas y por supuesto una correcta integración social. Se mencionan las condiciones que de cumplirse, pudiera avanzar mucho más y mejor en el proceso de hacer realidad UNA UTOPIA.

Palabras claves: Educación Especial; Inclusión; Educación; Desarrollo psíquico humano.

\section{Guillermo Arias Beatón}

Doutor em Ciências Pedagógicas

pelo Instituto Central de Ciências

Pedagógicas de Cuba - Cuba gariasbeaton@gmail.com

\section{Para citar este artigo:}

BEATÓN, Guillermo Arias. Condiciones y premisas para hacer realidad una utopia. Revista Linhas. Florianópolis, v. 16, n. 31, p. 08 - 25, maio/ago. 2015.

\section{DOI: $10.5965 / 1984723816312015008$}

http://dx.doi.org/10.5965/1984723816312015008 


\section{Conditions and assumptions to make a utopia}

\begin{abstract}
This article points out that the debate on the teaching of people with some kind of disability is not limited to the controversy Special School, School Integration or inclusion. The argument developed here shows that the real conditions and assumptions are not observed or never were fulfilled with regard to the teaching of these individuals, which is the true cause of the fact that these people do not reach the expected psychosocial development. It is the social and cultural conditions the true political and ideological causes that make children with disabilities do not learn and can not develop in a teaching model or another. This fight or discussion exists since antiquity to the present time and despite the progress made, they keep on resulting in poor quality of teaching, which does not make learning easier, the integral development of these people and of course the adequate social integration. If we had some of these conditions fulfilled, we could advance a lot and better in the process of making the utopia of quality teaching for these people.
\end{abstract}

Keywords: Special Education, Inclusion, Teaching, Human Psychic Development.

\section{Condições e pressupostos para fazer uma utopia}

\section{Resumo}

O presente artigo destaca que o debate sobre a educação das pessoas com algum tipo de deficiência, não resume-se apenas a controvérsia Escola Especial, Integração Escolar ou inclusão. $O$ argumento aqui desenvolvido apresenta que as reais condições e os pressupostos que não são cumpridos ou nunca foram plenamente satisfeitas no que tange a educação desses sujeitos, constituem as verdadeiras causas por que essas pessoas não atingem 0 desenvolvimento psicossocial esperado. São as condições sociais e culturais e até mesmo como parte deles, as verdadeiras causas políticas e ideológicas que fazem com que as crianças com deficiência não aprendam e não conseguem se desenvolver- em um modelo educativo ou outro. Esta luta ou discussão existe desde antiguidade até o momento atual e apesar dos progressos feitos, continuam a resultar em educação de má qualidade, o que não facilita a aprendizagem, o desenvolvimento integral dessas pessoas e, claro, a integração social adequada. Se tivéssemos algumas dessas condições cumpridas, poderia se avançar muito mais e melhor no processo de realizar a utopia da escolarização de qualidade para essas pessoas.

Palavras-chave: Educação Especial; Inclusão; Educação; Desenvolvimento psíquico humano. 


\section{Introducción}

El ser humano no puede vivir sin utopías, sin desafíos, proyectos y uno de ellos es llegar a lograr una verdadera y efectiva educación y formación de los escolares con necesidades educativas especiales. Mucho se ha logrado ya en este sentido, y el pasado siglo fue testigo de avances notorios. Sin embargo, aún se presentan muchas dificultades que hacen insuficiente, cualquier tipo de programa de atención y educación a estos escolares. Lamentablemente, aun ni la educación especial, ni los diferentes modelos de integración escolar y últimamente los denominados como inclusión, han demostrado la efectividad que pudiera y debiera tener el proceso de educación, aprendizajes, corrección, compensación y desarrollo de los escolares con algún tipo de déficit biológico o con necesidades educativas especiales.

El problema esencial, a mi juicio, no se encuentra en la polémica educación especial versus integración o inclusión, sino en la materialización de las siguientes premisas o condiciones que garanticen un proceso de educación y formación verdaderamente de calidad, orientado a proporcionarle a estas personas la educación, enseñanza y atención acorde con las exigencias y necesidades que el tipo de déficit biológico plantea a los contenidos sociales y culturales de nuestra sociedad de nuestra cultura y en definitiva a los contenidos y organización de nuestras escuelas.

Tampoco creo que resuelvan mucho los aspectos relacionados con la organización de los centros, los de carácter instrumental o de la cooperación pedagógica, que si bien todos son importantes, no son los que considero esenciales y en lo que se deberían trabajar para garantizar la educación de calidad para todos y en particular para los escolares con NEE. A continuación voy a analizar los aspectos que considero medulares e incluso que facilitarían la comprensión y aplicación de los otros más mencionados en la literatura especializada. 


\section{Desarrollo del contenido}

Una primer condición es, la adopción de una concepción integral y más próxima a la realidad, acerca de cómo se produce el desarrollo psicológico humano, y en particular, de los escolares con necesidades educativas especiales o con algún tipo de déficit biológico; en segundo lugar, garantizar un proceso de atención al desarrollo de los niños con riesgos en su desarrollo y a sus familiares desde las edades más tempranas y la preescolar; en tercer lugar, lograr que los familiares, educadores, maestros, profesores, funcionarios y políticos en general sean formados y preparados, fundamentalmente, en la concepción mencionada acerca del desarrollo, y para la atención a la diversidad y por último, trabajar por una mayor calidad en la labor de la escuela y del sistema de educación en general y que es de lo que más se escribe en la actualidad. Por supuesto, las tres primeras premisas y condiciones, facilitarían el logro de esta última.

En este sentido, si hacemos una revisión rápida acerca del estado actual de la calidad y efectividad de los sistemas de educación en muchos países del mundo, se pudiera suponer que los procesos integradores o de inclusión, no se realizan con la calidad necesaria.

Resulta paradójico, y hasta cierto punto contradictorio, que en países donde el 50 \% de los niños de la educación fundamental no aprenden a leer y a escribir, o donde el 30 \% de los que llegan al 6to grado, tampoco han adquirido estos preciados instrumentos y donde el $72 \%$ de los que llegan a la universidad, no comprenden lo que leen, la educación integrada o inclusiva de los escolares con necesidades educativas especiales, garantice, el aprendizaje de la lectura, la escritura, el cálculo y el resto de los contenidos de la enseñanza que sabemos que estos escolares pueden aprender para que se les produzca el desarrollo psíquico correspondiente.

¿Cómo es posible que podamos admitir que un programa de integración o inclusión pueda ser eficiente con tal situación general de los sistemas de educación, cuando el índice de los escolares con estas características se encuentra aproximadamente entre el 10\% y el 12\%, en los países donde ocurren estos hechos? ¿Es posible creer que si más del 30\% de los escolares no reciben una buena enseñanza, el 12\% de aquellos escolares que exigen una enseñanza más dedicada y orientada por una 
concepción optimista del desarrollo, o sea, una enseñanza que verdaderamente promueva el desarrollo infantil, que incluso lo compense y lo corrija, son atendidos educacionalmente como lo merecen o necesitan?

Lo más importante de esta utopía, lo esencial, es que lo que sabemos que se puede alcanzar con los niños con necesidades educativas especiales, que se logre, un aprendizaje y adquisición de los instrumentos y medios culturales producidos por el ser humano en milenios de producción de la cultura y de la sociedad humana, que les permitan, mediante la apropiación de estos contenidos sociales e históricos, la formación y el desarrollo de los procesos psíquicos superiores, así como una formación de la personalidad independiente, autónoma y autorregulada; que les garantice una adecuada calidad de vida y una inserción real en la vida social.

Lo más importante para mí, es reflexionar sobre este problema y no sobre la integración o inclusión versus la educación especial. Lo que propongo, cuando lo logremos, la inclusión se producirá como una resultado natural del proceso de desarrollo. Creo que la escuela para todos y de calidad, que garantice lo que anteriormente hemos delineados, debe ser un propósito de todas las personas y países del mundo. Creo en la posibilidad de la integración y la inclusión, en la atención a la diversidad, creo en una sociedad para todo, pero ella está por construirse. Sin embargo, en lo que pienso es si la escuela, mundialmente, y sobre todo, la sociedad en general y los sistemas nacionales de educación, están preparados para cumplir con las exigencias de una responsable educación a los escolares con necesidades educativas especiales.

El problema de la atención y educación a las personas con necesidades educativas especiales ha ido evolucionando, en la historia de la cultura, hacia posiciones francamente más optimistas (ARIAS, 2005); se conciben y aceptan más, las amplias posibilidades de aprendizaje y desarrollo que estas personas poseen, y se asume la diversidad de aprendizajes y desarrollo como lo verdaderamente normal en la población de seres humanos, aceptándose la diversidad como un valor y cualidad y no como motivo de desigualdades y exclusión. Todo ello ha conducido, y también es un producto, a la vez, de las tendencias progresistas de la integración escolar o inclusión de las personas con necesidades educativas especiales. Muchos preconceptos se han ido eliminando en la sociedad, gracias a estos movimientos de avanzada. Sin embargo, esto está muy lejos de 
ser una concepción generalizada y sobre todo que produzca sistemas educacionales y sociedades que resuelvan con la calidad necesaria, los desafíos que produce la atención educacional a estas personas.

La razón fundamental de esta evolución, a mi juicio, es una mayor conciencia y conocimiento de las fuerzas motrices y la génesis del desarrollo, así como del papel de la educación y la enseñanza en ello (VYGOTSKI, 1989, COMENIO, 1983). Los diferentes grupos de orientación humanista y progresista que han surgido en los diferentes países del mundo, han promovido este cambio. Los estudios realizados y las concepciones basadas en estas importantes relaciones han ido penetrando cada vez más, en la cultura acerca del ser humano, su formación y desarrollo.

No obstante estos avances, en las sociedades excluyentes continúan existiendo y desarrollándose posiciones biologicistas, fatalistas e innatistas, que siguen luchando, y aunque han perdido fuerza, se presentan enmascaradas en un preformismo y un no humanismo residual. Esto está dado por las insuficiencias de las explicaciones científicas sobre el desarrollo psicológico infantil, que no llegan a presentar, de manera adecuada, la interdependencia o mediación compleja y dinámica que se produce entre las condiciones biológicas, las socioculturales y las propiamente psicológicas que se van conformando en el desarrollo humano, lo que resulta una condición necesaria e imprescindible para que se produzca el desarrollo psicológico humano, con una orientación más optimista, integral y que permita orientar una educación emancipadora y no amarrada a una concepción biologicista rígida e inmutable, fatalista como todavía existe.

Todo esto es lo que produce, a mi juicio, que las prácticas educativas no sean de la calidad necesaria, porque sus sustentos teóricos, metodológicos y conceptuales, se basan en una concepción del desarrollo espontánea, que se produce desde lo interno del niño, y a través de sus mecanismos fisiológicos y neurofisiológicos, lo que nada o poco tiene que ver con una enseñanza verdaderamente desarrolladora, promovida por las condiciones y organización que realizan los maestros del contexto social y cultural, que se constituyen en el origen o fuente mediatizado del desarrollo psíquico, aunque este se produzca, en última instancia, a lo interno y de acuerdo con sus propias leyes, que por supuesto incluyen las regularidades que estamos mencionado con insistencia. Como estamos hablando de sujetos portadores de determinados déficit biológicos que pueden afectar 
su desarrollo psíquico, tenemos también que insistir en los sistemas epigenéticos, la plasticidad, la flexibilidad y complejidad dinámica del funcionamiento biológico (lo genético y lo neurofisiológico) para contrarrestar las concepciones fijas e inmutables de una biología mecanicista y determinista del desarrollo humano, que no existen en realidad (GOULD, 2007 LEWONTIN, ROSE Y KAMIN, 1987, CASANOVA 2015 Y PEDROL 2015).

Es como si todas las posibles causas: sociales, económicas, de la poca calidad de la práctica educativa, se sustituyeran por un proceso interno insuficiente del desarrollo infantil, por sus esquemas iniciales y posteriores, sin establecer una precisa y clara relación entre el desarrollo de estas estructuras y su funcionamiento, con todas las insuficiencias sociales y culturales que el proceso de la educación presenta en la sociedad, la escuela y por ende en la familia, que afecta al desarrollo psíquico humano. Por eso es la calidad de la educación la que en definitiva se constituye en la causa inicial y original que desencadena el proceso de formación y desarrollo de los procesos internos y funcionales que definitivamente o en última instancia, logra el desarrollo a lo interno del sujeto en su proceso de constitución como ser social, cultural e histórico.

No es el cerebro, que solo por medio de su propio o intrínseco funcionamiento, el que aprende y se desarrolla psíquicamente, no son los genes que constituyen el genoma humano los que, por sí mismo, garantizan el desarrollo de las estructuras y el funcionamiento de lo subjetivo o desarrollo psíquico humano, son las condiciones sociales y culturales, que son históricas, las que impactando sobre lo uno y lo otro, en estrecha relación con el funcionamiento genético y neurofisiológico, lo que permite que se produzca la formación y desarrollo de las estructuras, el contenido y el funcionamiento específicamente humano, su subjetividad, su manera de hablar, de pensar y de actuar.

A mi juicio, por todo lo mencionado, también hay otras causas, que llegan a ser fundamentales, de orden político e ideológico, las que producen los inadecuados aprendizajes y los insuficientes desarrollo neurofisiológicos y psíquicos. Me refiero a la dificultad, casi intrínseca, de la psicología cognitiva y demás ciencias médicas, biológicas y sociales, en el reconocimiento y aceptación de que son los factores sociales y culturales los que, definitivamente y en realidad, producen los males y trastornos en el desarrollo psicológico y social del ser humano. Fuertes grupos progresistas han venido luchando 
desde épocas tempranas en estas sociedades para contrarrestar los efectos de esta ceguera política, que frena también a las ciencias (VYGOTSKI, 1987 LEWONTIN, ROSE, KAMIN, 1987, GOULD, 2007, TOBACH, 1999, ARIAS, 2005b, 2006, CASANOVA, 2015 Y PEDROL, 2015).

Otra concepción teórica y metodológica requiere una educación y una escuela llamada a lograr la integración escolar y la inclusión de los niños con necesidades educativas especiales: La enseñanza debe producir y anteceder al desarrollo, apoyándose en él, pero promoviéndolo, haciendo que se formen nuevas y más complejas estructuras psicológicas superiores, como consecuencia de un adecuado y efectivo aprendizaje de los objetos, instrumentos y medios esenciales de la cultura. Este aprendizaje depende de la calidad de la enseñanza que organicen y dirijan los familiares, los maestros y toda la sociedad en general (VYGOTSKI, 1996, 1988, 2001).

En este sentido, las investigaciones que hemos realizado, demuestran el papel esencial que juegan las concepciones del desarrollo que poseen las madres, los padres y maestros en su desempeño como educadores y promotores del desarrollo infantil, que explicaré posteriormente (ARIAS, 2001).

Otra premisa esencial para garantizar una efectiva integración escolar de los niños con algún tipo de necesidad educativa especial es la de lograr, lo más temprano posible, una atención preventiva y promotora del desarrollo en este tipo de niño. Una forma eficiente de lograr este propósito, es trabajar con la noción de riesgo para el desarrollo infantil.

Hoy en día, desde el punto de vista epidemiológico, es reconocido que existen niños en situación de riesgo para su desarrollo (BRICKER, 1991). Estos riesgos, aunque no son necesariamente causas directas de dificultades en el desarrollo infantil, las pueden producir, cuando determinadas condiciones insuficientes de naturaleza social y cultural se presentan y actúan y no se logran contrarrestar o eliminar los efectos que dichas condiciones o riesgos pueden llegar a producir en el aprendizaje y el desarrollo psíquico humano. Se conoce también que, estos riesgos se pueden presentar en un sistema y plurideterminadamente. 
Estas condiciones, el valorar que tienen es que permiten realizar una labor preventiva encaminada a evitar sus efectos mediante una intervención social y cultural adecuada. Descubrir estos riesgos y atender que no produzcan los efectos que ellos pueden producir, es una manera positiva de enfrentarlos y se constituyen en indicadores para ejercer una atención y una educación preventiva, encaminada a evitar insuficiencias en el desarrollo infantil, o a promover su compensación. Los posibles riesgos se pueden encontrar, en un alto por ciento, en sujetos con posibilidades de tener déficit biológico o condiciones sociales y culturales desfavorables, por eso se encuentran como antecedentes, en la mayoría de los escolares que se conocen como personas con necesidades educativas especiales.

La apreciación de estos riesgos no es hacer un diagnóstico, estos solo aportan indicadores para realizar acciones encaminadas a evitar que el desarrollo psíquico inadecuado o insuficiente se constituya o instaure en el sujeto. La educación infantil desde la más temprana edad es la forma ideal y más adecuada para garantizar eliminar los riesgos que presenten algunos niños y niñas en su desarrollo (ARIAS, 2006).

Desde la década de los 60, la atención y educación temprana a los niños con riesgos para su desarrollo y sus familiares, se ha constituido en una labor de alto valor social y científico, debido a que puede facilitar que el proceso de desarrollo logre una mayor efectividad en su curso y evitar que surjan algunas de las desviaciones o alteraciones que se producen en los escolares llamados con necesidades educativas especiales.

Esta labor, además de lograr un mejor desarrollo infantil, permite obtener datos importantes acerca del conocimiento de cómo este desarrollo se produce y de cómo se pueden prevenir sus alteraciones a través de acciones sociales y culturales, constituyéndose en una condición fundamental que garantizaría una correcta integración escolar, inclusión y de hecho una educación especial, más efectiva para los niños con necesidades educativas especiales, en el contexto de la escuela regular.

Los trabajos e intentos reformistas que se realizaron en la década de los 60 en los Estados Unidos con la Educación Remedial, son un ejemplo de ello. Lo que se realiza en Cuba desde la organización integral de todo el Sistema Nacional de Educación, desde las 
edades iniciales desde los años 70s y 8os hasta nuestros días, también es un intento y una evidencia para garantizar este proceso del que hablamos.

Los educandos de esta manera arriban a la educación primaria, básica o fundamental con el desarrollo real necesario para recibir una enseñanza o tareas de mayor complejidad, que con la manera en que se organice por parte del profesor o maestro y se brinden las ayudas correspondientes, permitirá nuevos aprendizajes y superiores desarrollos de las funciones psíquicas superiores o de naturaleza cultural. Así funciona el desarrollo arrastrado o halado por la educación de calidad que su artífice, el educador, se lo proponga conscientemente, organizando el contexto social y cultural que necesita el escolar para aprender y desarrollarse.

En la medida en que los niños con riesgo para su desarrollo o con necesidades educativas especiales lleguen a la escuela primaria, en posesión de un desarrollo que les permita una mayor autonomía e integración, entonces se facilitará todo tipo de enseñanza y educación, a estos, en el contexto de la escuela regular, y a su vez, la educación permitirá promover con mayor efectividad su desarrollo futuro.

En los estudios realizados por nosotros, se evidencia que los riesgos más complejos de atender y que influyen más negativamente en el desarrollo infantil son: el abandono familiar y social, el desconocimiento por parte de las madres y los padres sobre qué hacer como educadores de sus hijos y la existencia en las madres o en los padres de conflictos emocionales no resueltos con la educación en la familia de origen.

Lo más grave es que estos problemas que denominamos como los verdaderos riesgos para el desarrollo infantil, se presentan de forma conjunta e interrelacionada, produciéndose, en la mayoría de los casos, el abandono familiar y social desde el punto de vista educativo y desarrollador.

Las influencias negativas de estos riesgos, pueden compensarse, en la medida en que se logre un trabajo de orientación con la familia, que garantice, que en ella se realicen acciones educativas, estimuladoras y promotoras del desarrollo infantil. En nuestras investigaciones hemos comprobado que con este tipo de labor, se puede llegar a lograr que la inmensa mayoría de los padres orientados, se constituyan en promotores del 
desarrollo infantil, y como resultado, sus hijos, lleguen a alcanzar niveles adecuados en su desarrollo integral.

Estos padres realizan cotidianamente tareas para estimular el desarrollo de sus hijos, que por su globalidad y por el contexto afectivo y de interrelaciones en el que se producen, incluyen una serie de actos y acciones importantes que van permitiendo un desarrollo sostenido de la psicomotricidad, la percepción y formación de representaciones y modos de actuar, de la memoria, el lenguaje oral, el pensamiento y demás procesos y funciones psíquicas superiores específicas. Este desarrollo se constituirá, en la base para la formación de otros sistemas psicológicos complejos, como el dominio de la lectura, la escritura, el cálculo, las leyes de la historia, la concepción del mundo, la autovaloración y la autorregulación, entre otros, que posteriormente tendrá lugar producto de la labor educativa en la escuela (VYGOTSKI, 1991).

Conjuntamente con estas acciones es imprescindible que se cree un ambiente emocional positivo en el sistema de interrelaciones, que todo lo que se produzca sea vivenciado por el niño positivamente, presentándose las normas de convivencia social de manera suave e imperceptiblemente, de forma tal que vayan siendo aceptadas por la propia conveniencia, utilidad y el propio interés y deseo del niño (BOZHOVICH, 1976).

Los padres y madres potenciadores o promotores del desarrollo infantil adecuado, poseen una comprensión de las características del niño y un conocimiento del nivel de desarrollo que este posee, así como, del que se puede ir promoviendo a través de variadas y múltiples acciones educativas (ARIAS, 2001, 2006). Las características que hemos detectado, en los estudios mencionados, en los familiares potenciadores del desarrollo son:

- Poseen una creencia o concepción adecuada de cómo se produce el desarrollo infantil, atribuyéndole a la familia un papel central en la organización de una vida familia que facilite el aprendizaje y el desarrollo de sus hijos.

- Asumen un papel activo en la educación infantil, promoviendo y organizando acciones que conduzcan a formar las cualidades que desean que sus hijos posean.

- Comprenden el papel de otros agentes sociales, educacionales y culturales y colaboran e interactúan de manera comprometida con ellos. 
- Poseen una intencionalidad en la búsqueda de herramientas o instrumentos para realizar su papel con efectividad.

En estos casos, las formulaciones personales acerca de como consideraban que se producía el desarrollo infantil, se aproximaba más al que se formula en el enfoque histórico-cultural o sea, que el desarrollo psicológico del niño es esencialmente de origen social y cultural y que depende mucho de lo que los padres logren estimularlo y organizar la educación en el seno de la familia; que los factores biológicos son importantes y por eso la necesidad de una buena alimentación y atención higiénico -sanitaria, pero estableciendo una clara e implícita diferencia entre un desarrollo y otro, aunque existiera una interacción o mediación. Conscientemente atribuyen el desarrollo a la labor educativa y no solo a la maduración biológica.

Hemos podido apreciar, también, que el elevar el nivel de instrucción de una población, puede llegar a ser una condición necesaria para lograr lo antes mencionado, pero ello no es suficiente y hay que trabajar por preparar a la familia y la escuela en tan importante actividad. Para ello es necesario preparar a la familia y toda la sociedad en una concepción acerca del desarrollo psicológico infantil y que lleguen a dominar las explicaciones de las fuentes, génesis y dinámica de ese desarrollo, precisando el papel que tienen los padres, madres, coetáneos, maestros y toda la sociedad en general, en este proceso de desarrollo infantil, de preparación del niño para la escuela y de su desenvolvimiento escolar.

Algo semejante se presentó en otro estudio que se realizó con los denominados maestros potenciadores y no potenciadores, donde se llegó a determinar que las características que definían o diferenciaban mejor a los primeros de los segundos, eran las siguientes:

- Concepción del desarrollo infantil más relacionada con las condiciones socio, histórico, cultural del contexto donde se desenvuelven.

- Se preocupan por adquirir los instrumentos para potenciar el desarrollo infantil. Relacionan más estrechamente sus estudios de posgrado con esta necesidad.

- Insisten mucho más en atender y promover el desarrollo intelectual y afectivo de los niños de manera integral. 
- Dominio y mayor flexibilidad en la aplicación de los programas de la enseñanza, atendiendo a los intereses y necesidades de los escolares.

- Significado positivo del proceso de formación pedagógica y su práctica profesional.

Como puede apreciarse los educadores que mejores resultados obtienen en el trabajo educativo, y en particular en el desarrollo del escolar, son aquellos que, al igual que los familiares, poseen una mejor explicación y comprensión de cómo se produce el desarrollo psicológico infantil y la labor que hay que realizar para lograrlo.

Otro tipo de atención que debe producirse conjuntamente con la labor educativa de la familia, es la Educación infantil o Educación inicial y preescolar en Instituciones donde especialista y profesionales de una preparación esmerada, no solo contribuyan con la educación y desarrollo de los niños en riesgos en su desarrollo, incluyendo los que pudieran portar déficit biológicos sino también, que promuevan la formación que los miembros de las familias necesitan para poder colaborar plena y conscientemente en la educación y desarrollo de dichos sujetos.

Si los niños y niñas con una dinámica del desarrollo psíquico adecuada precisan de la Educación Inicial y preescolar que promueva su desarrollo y su educación; aquellos en situación de riesgo social y cultural e incluso portadores de algún déficit o problema biológico, lo necesitan mucho más.

Partimos en este trabajo de la concepción que adopta lo Histórico Cultural sobre el desarrollo psíquico humano, que considera que este, es de naturaleza cultural, a diferencia del que ocurre en los seres vivos anteriores al ser humano, que es de naturaleza biológica. Ese desarrollo eminentemente humano, se consigue por medio de las relaciones sociales, interpersonales o intersubjetivas que son portadoras de los contenidos de la cultura (objetos y medios culturales) producidos por el ser humano a todo lo largo de la historia de la sociedad. En este aspecto también la diferencia con los seres vivos anteriores al ser humano, es que estos últimos establecen interacciones de naturaleza biológica, porque solo reaccionan de manera instintiva según los logros que le proporciona la evolución de la especie, alcanzándose la adaptación de una manera fija y siempre por la misma forma de comportamiento. 
Por esta razón, en el ser humano, es en la educación donde se organizan y realizan en la familia, la escuela o centros educativos y en la sociedad, los contenidos y medios de la cultura y las diferentes formas de relaciones sociales, las actividades y la comunicación, que permiten los aprendizajes y el desarrollo psíquico del ser humano, el desarrollo cultural de los contenidos de la subjetividad.

Todo este proceso ha de promover en los niños, un buen desarrollo y comprensión del lenguaje oral para la comunicación entre las niñas y los adultos, una apropiación de los objetos, en cuanto a su uso y denominación. El dominio del empleo de objetos instrumentos para la solución de tareas o problemas prácticos; la adecuada realización del dibujo y el juego, principalmente el de papeles, la modelación a partir de representaciones gráfica o por medio de la imaginación y por último, el lograr que los niños adquieran la conciencia de que el lenguaje hablado se realiza por medio de palabras que a su vez están constituidas por diversos tipos de sonidos y que representan a los objetos de la realidad; pudiéndose dicha palabras y sonidos representar de diferentes formas materiales o gráficas.

Todo este procedimiento cultural, social y educativo resulta muy importante que los niños y niñas lo reciban en las instituciones de Educación inicial y preescolar, pero resulta imprescindible, con sus ajustes específicos, para aquellos que son portadores de riesgos sociales, culturales o déficit biológico, como medio para evitar que el riesgo que portan, se apodere de su desarrollo psíquico y lo frene o lo impida. Solo trabajando por eliminar los efectos o compensar los daños que estos riesgos producen, es que podemos garantizar que el desarrollo de estos educando, sea todo lo mejor que puede llegar a ser.

\section{Conclusiones}

Por todo esto que hemos analizado consideramos que la educación en la familia y la Educación Infantil o Inicial y Preescolar, que apoya y educa también a la familia, se constituyen en una condición esencial para lograr el desarrollo real necesario con el que deben llegar a la escuela o educación fundamental los educandos con algún tipo de 
déficit biológico o condiciones sociales y culturales desfavorables (riesgos) y puedan continuar sus estudios de manera incluida en la escuela para todos y de calidad.

Este análisis responde al hecho que prácticamente nadie discute de que en las edades más tempranas es mucho más conveniente contribuir a promover y compensar el desarrollo psíquico de aquellos sujetos portadores de riesgos sociales y culturales para su desarrollo y con déficit biológicos. Esta condición si se logra, no elimina la necesidad de que las escuelas tengan la posibilidad de recibir servicios especializados para aquellos estudiantes con las condiciones que estamos analizando, que lo necesiten y los profesores estén preparados para continuar solucionando y compensando los efectos que dichos riesgos o déficit biológicos pudieran continuar produciendo.

Estos resultados nos hacen insistir en la necesidad del debate de los problemas del desarrollo psicológico infantil, desde una perspectiva histórico-cultural, y de promover que familiares, maestros, educadores y profesores sean preparados en este tipo de concepción, porque ella parece producir una base orientadora positiva a la labor que estos adultos deben realizar para estimular y potenciar dicho desarrollo.

El proceso de integración o inclusión de los niños con necesidades educativas especiales así como una educación especial eficiente, exigen que las premisas o condiciones antes señaladas se logren cumplir, y con ello tenga lugar un sistema educativo que garantice un aprendizaje de los instrumentos culturales esenciales tales como la lectura, la escritura, el cálculo, y demás conocimientos, y también, de los demás contenidos de la formación integral de su personalidad.

El contenido de las reflexiones realizadas, me hace concluir este análisis con una idea de L.S. Vygotski en los años 20 del siglo pasado, que él no explicó mucho, pero que yo interpreto, quizás como la pretendió a interpretar él, ochenta años después. Vygotski dijo:

La enseñanza conjunta con los niños normales que se ha planteado en múltiples ocasiones por Grazer y otros, ahora, lamentablemente, no puede ser todavía un problema del orden más próximo, pero la consigna de Grazer es también nuestra consigna. (VYGOTSKI, 1989 p. 69) 
Estas palabras, a mi juicio, encierran un optimismo y una posibilidad de lograr la integración escolar o la inclusión que muchos queremos, pero para lo cual es necesario un debate más sistemático de los problemas sociales, culturales, económicos y científicos en la sociedad. El problema no se resolverá haciendo la integración a la fuerza o por decreto o con el discurso de la inclusión, que permitirá que haciéndola, ella vaya creando las condiciones; ello puede afectar el verdadero desarrollo que puede llegarse a producirse en las personas con necesidades educativas especiales, y con ello puede llegar a desanimar, a hacer perder la confianza en las fuerzas de la educación y la enseñanza en el desarrollo humano, a producir más pesimismo, y peor aún, a brindarle argumentos a científicos como un Jensen, psicólogo de la prestigiosa Universidad de Harvard, que en los años finales de los 60s, presentó una argumentación biologicista, reaccionaria, fascista y anticientífica basada en los errores de proyección y fundamentación del progresista movimiento de la Educación Remedial en los Estados Unidos.

Es necesaria una reflexión y una sistematización científica y mancomunada, sin búsqueda de protagonismos, sin acusaciones, simplemente con un gran deseo, y por supuesto, con una gran pasión: HACER REALIDAD UNA UTOPÍA. 


\section{Bibliografía}

ARIAS BEATON, Guilhermo. La Educación Especial en Cuba. Su desarrollo y perspectivas. Conferencia Especial. Congreso PEDAGOGIA'86, 1986. Materiales del Congreso.

ARIAS BEATON, Guilhermo. Evaluación y Diagnóstico en la Educación y el Desarrollo. Desde el Enfoque Histórico Cultural. Edición Laura Calejon, Sao Paulo. 2001.

ARIAS BEATON, Guilhermo. Desarrollo histórico de los escolares con necesidades educativas especiales. Apuntes y consideraciones. En Psicología Especial Tomo I. Colectivo de Autores. Editorial Félix Varela, Ciudad de la Habana, Cuba, 2005.

ARIAS BEATON, Guilhermo. La Persona en el Enfoque Histórico Cultural. Editora Linear B, São Paulo, Brasil, 2005b.

ARIAS BEATON, Guilhermo. Inteligência e educação. Editorial Terceira Margem, São Paulo, Brasil. 2006.

BOZHOVICH, Lidija I. La personalidad y su formación en la edad infantil. Editorial Pueblo y Educación, La Habana, 1976.

BRICKER, Diane. Educación temprana de niños en riesgos y disminuidos. Editorial Trillas, México, 1991.

CASANOVA PERDOMO, Ana Rosa. Epigenética: contenido esencial para comprender el desarrollo psicológico en el hombre. En Alternativas Cubanas en Psicología, Volumen 3. Número 7/2015 pp. 53-63. La Habana Cuba, 2015.

COMENIO, Johann Amòs. Didáctica Magna. Editorial Pueblo y Educación. Ciudad de la Habana, Cuba, 1983.

GOULD, Stephen Jay. The Richness of Life. The essential Stephen Jay Gould. Edited by Steven Rose. W.W. Norton \& Company, New York, 2007.

JENSEN, Arthur Robert. How Much Can We Boost IQ and Scholastic Achievement? In Harvard Educational Review Vol. 39 No. 1, 1969.

LEWONTIN, Richard Charles, Steven, ROSE Y KAMIN, Leon. No está en los genes. Ed. Crítica, Barcelona. 1987. 
PEDROL TROITEIRO, Rina. Determinismo biológico vs determinismo mediado en el desarrollo humano. En Alternativas Cubanas en Psicología, Volumen 3. Número 7 / 2015 pp. 42-52. La Habana Cuba, 2015.

TOBACH, Ethel. Evolution, Genetics and Psychology: The Crisis in Psychology. Vygotsky, Luria and Leontiev Revisited. In the book Activity Theory and Social Practice Edited by Seth Chaiklin, Mariane Hedegaard and Uffe Juul Jensen. AARHUS UNIVERSITY PRESS. 1999.

VYGOTSKI, Lev Semenovitch. Interacción entre enseñanza y desarrollo. En el libro Selección de Lecturas de Psicología de las Edades I. Colectivo de Autores, Facultad de Psicología Universidad de La Habana, Editorial del Ministerio de Educación superior, 1988.

VYGOTSKI, Lev Semenovitch. Fundamentos de Defectología, Tomo V. Editorial Pueblo y Educación. La Habana, 1989.

VYGOTSKI, Lev Semenovitch. Obras Escogidas, Tomo I Editorial Aprendizaje/Visor, Madrid, España, 1991.

VYGOTSKI, Lev Semenovitch. Obras Escogidas, Tomo IV. Editorial Aprendizaje/Visor, Madrid, España. 1996.

VYGOTSKI, Lev Semenovitch. Psicología Pedagógica. Un curso breve. Aique grupo Editor S.A. Buenos Aires, Argentina. 2001. 